\title{
Kurze Bemerkung über das Führungsfeld der Quantenmechanik.
}

\author{
Von P. Gruner in Bern.
}

(Eingegangen am 22. Januar 1927.)

$\mathrm{Zu}$ den wertvollen neuen Axbeiten Borns ${ }^{1}$ ), die einen Zusammen schlub der Heisenberg-Born-Jordanschen mit der de Broglie Schrödingerschen Quantenmechanik nach ganz neuen Gesichtspunkte: ergeben, erlaube ich mir folgende kurze Bemerkung.

Nach dieser neuen Bornschen Auffassung sind die Bewegungsgesetz der Materie zurückzuführen auf die rein mathematischen, abstrakt geltende: "Führungsgesetze", welche - ohne Energie zu besitzen - durch di Schrödingersche Differentialgleichung bestimmt sind, und durch welch die mit Energie und Impuls begabten "Korpuskeln" (Elektronen, Pro tonen oder wohl auch Lichtquanten) nach den Gesetzen der Wahrschein lichkeit bewegt werden: „Der Einzelprozeß ... ist also nicht kausa festgelegt, wohl aber die a-priori-Wahrscheinlichkeit seines Auftretens.

Ich möchte darauf hinweisen, da ich, allerdings in gänzlich anderer Zusammenhang, ähnliche Gedanken in einem Referat an der schweizerische: physikalischen Gesellschaft in Aarau, 9. August 1925 (Archives d. science phys, et natur. (5) 7, 342), geäußert habe. In diesem Vortrag sucht ich an Hand des Thomsonschen Modells der Lichtquanten (das wob nicht aufrechterhalten werden kann, und worauf es auch nicht ankommt den grundsätzlichen Gedanken festzulegen, dab die vorhandenen elektrc magnetischen Ladungen, d. h. die vorhandene Materie, ein Feld als rei geometrisches energieloses Führungsfeld bestimmen, und daß die tał sächliche Bewegung der Lichtquanten nach Wahrscheinlichkeitsgesetze erfolge. - Die Entdeckung der Schrödingerschen Differentialgleichun. und deren genialen Ausbau durch Born scheinen gerade auf der Linie $z$ liegen, die ich damals skizzierte, ohne selber imstande zu sein, sie wirk lich auszuführen.

Bern, Januar 1927.

1) ZS. f. Phys. 37, 863; 38, 803; 40, 167, 1926. 